\title{
SUPPRESSION OF STIMULATED RAMAN SCATTERING IN A TWO-COLOR THREE-BEAM SETUP
}

\author{
Thomas Würthwein ${ }^{1}$, Niels Irwin' ${ }^{1}$, Carsten Fallnich ${ }^{1,2}$ \\ 1. Institute of Applied Physics, University of Münster, Corrensstr. 2, 48149 Münster, \\ Germany \\ 2. MESA+ Institute of Nanotechnology, University of Twente, Enschede 7500 AE, The \\ Netherlands \\ E-mail: t.wuerthwein@uni-muenster.de
}

KEY WORDS: stimulated Raman scattering, suppression of stimulated Raman Scattering

Sub-diffraction limited imaging schemes have become widely used in fluorescence microscopy. However, the need for labeling with fluorescent dyes remains a major downside of fluorescence microscopy. In contrast, Raman imaging is inherently label-free. Unfortunately, the superresolution schemes used in fluorescence microscopy are currently not transferable to Raman microscopy. This paper presents the development of a scheme for the suppression of coherent Raman scattering through the depletion of probe photons, inspired by the work of M. Cho et al. $[1,2]$, who showed the suppression of coherent Raman scattering using laser pulses at three different wavelengths and two Raman resonances. In our case, probe depletion is achieved through saturated stimulated Raman scattering in a three-beam setup with only two colors involved. In the unsuppressed case, the combination of pump and probe pulse induces stimulated Raman scattering at a Raman resonance, leading to stimulated Raman loss of the probe and stimulated Raman gain of the pump. In the suppressed case a second strong pump pulse, acting as a depletion pulse, depletes the probe pulse via stimulated Raman scattering and induces a shortage of the probe photons at

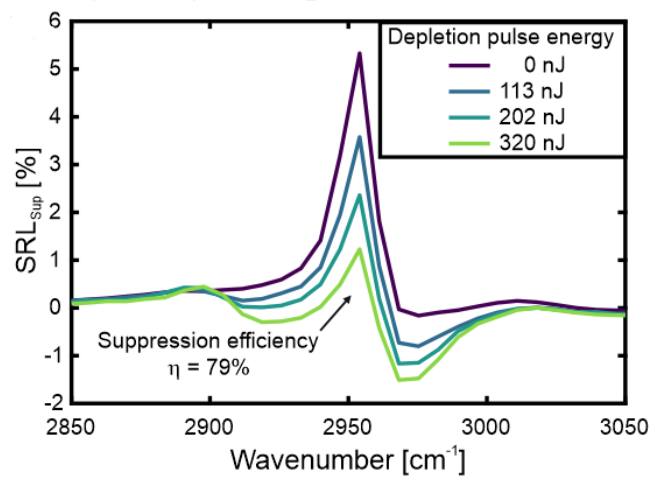
Figure 1: Suppressed Raman spectra in the $\mathrm{CH}$ strech region of acetonitrile. the Raman resonance, saturating the stimulated Raman scattering. Thus, only a small amount of additional SRLsup is induced by the original pump. If SRL is detected in addition to the spectral change, induced by the depletion pulse, the SRL will be reduced resulting in SRLsup $<$ SRL. Fig. 1 shows the stimulated Raman spectra of the $\mathrm{CH}$-stretch region of acetonitrile for different depletion pulse energies and a constant pump pulse energy of $60 \mathrm{~nJ}$. A reduction of the Raman signal at the resonance of up to $79 \%$ could be measured. The developed suppression technique is suitable to enable resolution enhancement in coherent Raman microscopy similar to stimulated emission depletion (STED) and opens up a simplified pathway towards label-free and subdiffraction limited imaging in comparison to references [1, 2].

[1] M. Cho, “Three-beam double stimulated Raman scatterings,” J. Chem. Phys 148, 014201, (2018).

[2] D. Kim, D.S. Choi, J. Kwon, S.-H. Shim, H. Rhee and M. Cho, "Selective Suppression of Stimulated Raman Scattering with Another Competing Stimulated Raman Scattering,” J. Phys. Chem. Lett 8, 6118- 6123, (2017). 